\title{
Optimal design of tapered roller bearings for maximum rating life under combined loads
}

\author{
EUGENIO DRAGONI ${ }^{\mathrm{a}}$ \\ Dipartimento di Scienze e Metodi dell'Ingegneria, Università degli Studi di Modena e Reggio Emilia, Via Amendola 2, \\ 42122 Reggio Emilia, Italy
}

Received 13 September 2015, Accepted 20 March 2016

\begin{abstract}
Using the relationships of the ISO 281 standard, this paper optimizes the internal dimensions of tapered roller bearings for maximum rating life. The bearing system addressed contains two identical bearings subjected to an arbitrary combination of centred radial and axial forces. It is shown that the basic rating life increases more than quadratically with the roller infill and the aspect ratio of the rollers, increases with the sixth power of the pitch diameter of the roller set and decreases with the third power of the applied radial force. Further, for any given ratio of axial to radial force, an optimal contact angle exists which maximizes the rating life of the bearing pair, irrespective of the actual bearing size and ratio of roller diameter to pitch diameter. The optimization procedure can either be used to design custommade bearings or to select from manufacturers' catalogues the bearing with the best contact angle for any assigned loading condition.
\end{abstract}

Key words: Radial rolling bearings / tapered rollers / rating life / design optimization

\section{Introduction}

Ordinarily, rolling bearings are not designed and built in-house but are chosen by the designer from the catalogue of specialized manufactures. The high degree of specialization has fostered the development of standardized high-quality products, readily available off-the-shelf in a wide range of shape and dimensions at affordable prices. Under particular design circumstances, like very large bearings or tight mounting spaces, the need can arise for non-standard bearings which the regular market can satisfy only at a considerable cost of time and money. In such instances, rolling bearings of simple geometry (as with cylindrical or tapered rollers) can be manufactured by the end user itself to meet the specific requirements at a fraction of the costs and delivery time requested by the specialized suppliers.

When tackling the construction of custom bearings the designer has the control of all the variables and the design is conveniently conducted according to optimization methods. Unlike conventional machine elements, for which a wealth of optimization criteria have been developed since long $[1,2]$, the category of rolling bearings has received so far relatively little attention. May be due to the aforementioned passive design approach (selection

\footnotetext{
${ }^{a}$ Corresponding author:

eugenio.dragoni@unimore.it
}

from a catalogue) towards these components, until the turn of the century the technical literature has been limited to optimal bearing selectors [3] and simulators of bearing kinematics [4]. Papers dealing with the optimization of bearing features have appeared only lately, aimed at maximizing one or several performance properties of ball bearings [5-11], cylindrical roller bearings [12,13] and tapered roller bearings [14-19]. For a detailed review of the literature on ball and cylindrical roller bearing optimization see $[20,21]$.

A pioneering instance of tapered roller bearing optimization can be traced back to the paper by Parker et al. [14], in which the performance of several large-bore (about $120 \mathrm{~mm}$ ), tapered roller bearings were simulated and tested at shaft speeds up to $20000 \mathrm{rpm}$ under combined thrust and radial load. The computer-optimized bearing design proved superior to equal-sized standard bearings tested for comparison. Chaturbhuj et al. [15] optimized tapered roller bearings using genetic algorithms and demonstrated that the fatigue life of the bearing improved marginally compared with respect to standard bearings. However, some authors $[12,13]$ have pointed out that some optimization constraints introduced in this paper were unrealistic. A method for optimizing the geometry of tapered roller bearings at high speeds was developed by Walker [16] with the main aim of determining the cup and cone angles which minimize the contact stresses 


\section{Nomenclature}

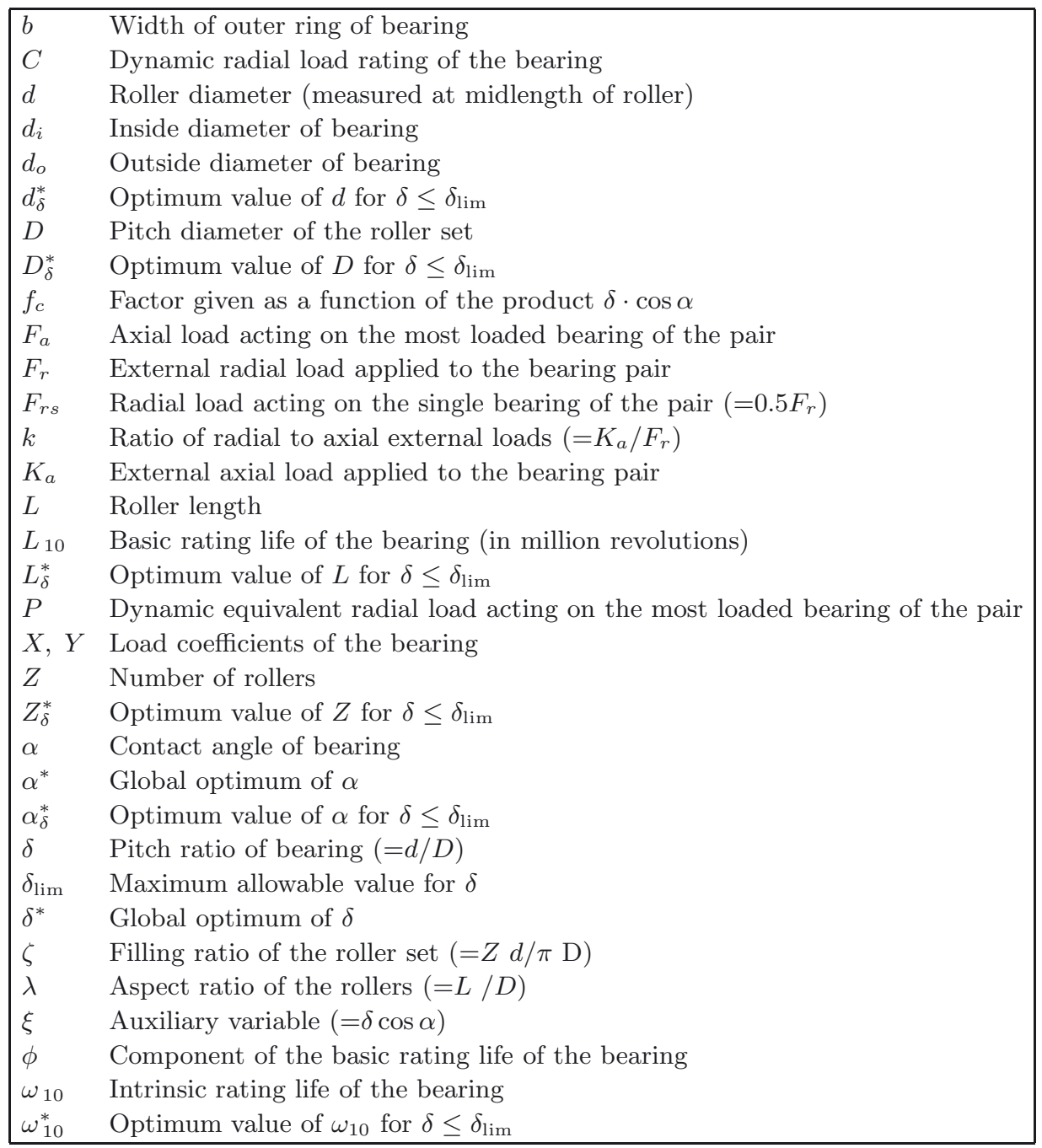

under a specific ratio of axial to radial load. Walker found that at low speeds the optimum cup angle is $40^{\circ}$, whereas the optimum value decreases to $10^{\circ}$ for the highest speeds. Wang et al. [17] presented a mathematical model for optimizing the design of four-column bearings with tapered rollers subject to several geometric constraints. By acting on a rearrangement of the classical variables (roller diameter and length, pitch diameter, number of rollers and cup angle), they improved the dynamic load rating by $22 \%$ and the life expectancy by $85 \%$ over a commercial competitor of like dimensions. Though interesting for the potential of improvements it shows, Wang et al.'s paper [17] does not provide details on the optimization method behind the model and little can be taken away from the published results apart from the specific example presented. Two comprehensive contributions to the optimal design of tapered roller bearings have recently been published by Tiwari et al. $[18,19]$. These two papers contain also excellent reviews of the technical literature on rolling bearings and the various optimization methods applied so far to bearing design.
In general, the above papers are focused on the methodological approach to the optimization problem and pay much less attention to the engineering merit of the optimization results. Most of the numerical algorithms referenced above can surely be beneficial to the specialist's work, but they are of little use for the generalpurpose mechanical designer confronted with the task of designing simple custom bearings or selecting the optimal bearing from a manufacture's catalogue. As outcome of an applied research for a small Italian manufacturer of planetary gear drives, the present author has recently published an optimization procedure for radial cylindrical roller bearings [20], which overcomes these limitations. Relying on easy step-by-step calculations and with no need for specific optimization backgrounds, that procedure gives the macro-geometry of the bearing (roller diameter, roller length, pitch diameter of roller set, number of rollers) which maximizes the static and the dynamic load ratings under realistic size constraints. A later paper by the author [21] has extended the research to radial bearings with tapered rollers under radial and axial static 


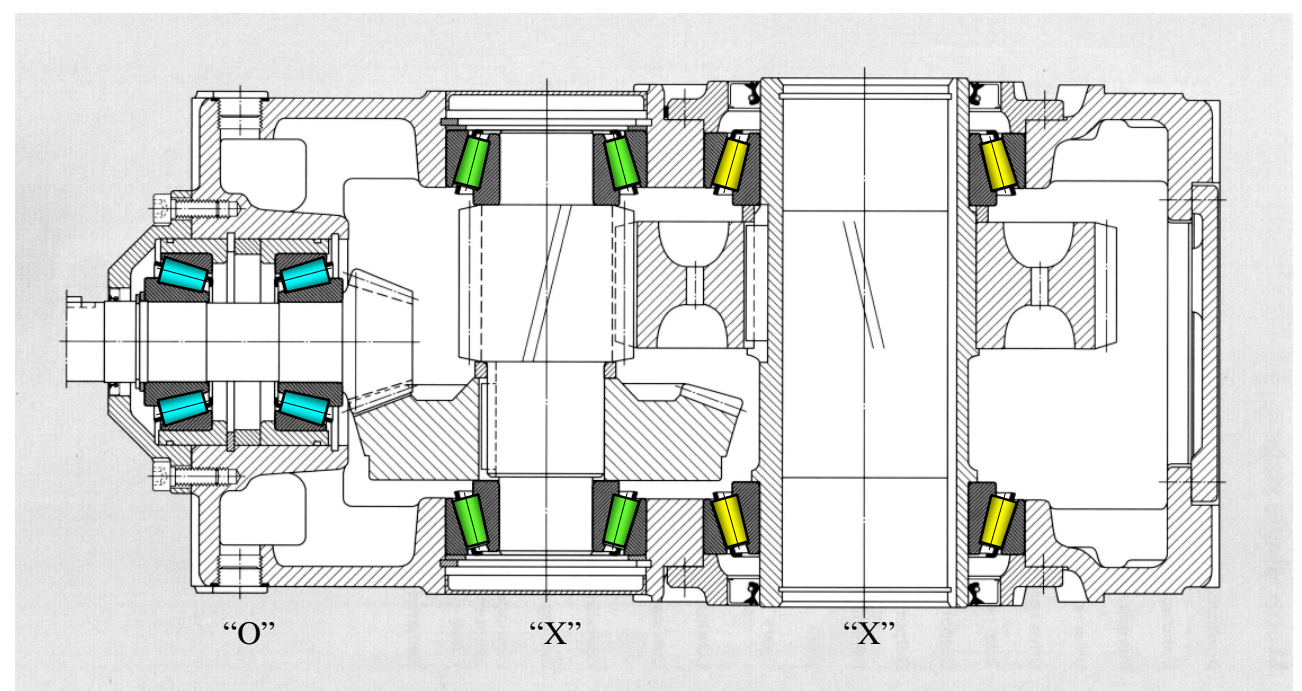

Fig. 1. Longitudinal section of a two-stage bevel/spur gearbox with "O" and "X" arranged tapered roller bearings susceptible of customization and/or optimization.

loads, showing that for any given ratio of axial to radial forces an optimal contact angle exists which maximizes the static capacity of the bearing system.

In the wake of that fruitful research, this paper tackles the optimization of tapered roller bearings for maximum dynamic capacity under an arbitrary combination of radial and axial loads. The optimization involves twin bearing pairs mounted according to either "O" or " $\mathrm{X}$ " arrangements as shown in Figure 1 for a typical application. Following the equations provided by the standard ISO 281 [22], the intrinsic rating life of the bearing system is expressed in terms of three parameters: the ratio between roller diameter and pitch diameter, the cup angle and the ratio of axial to radial force. This simple expression gives engineering insight into the problem and shows that for any given load ratio the rating life of the bearing is maximized by an optimal contact angle, nearly independent from the bearing size and proportions. Tables of optimal contact angles are provided for quick reference.

The optimization method presented here gives its best results for custom-made bearings, for which the design parameters can be varied with the greatest freedom. However, the results disclosed are useful also for identifying the best commercial bearings that can be selected from the manufacturers' catalogues to fit a particular application.

\section{Problem statement}

Figure 2 shows the baseline configuration of the bearing system examined in this paper. The two bearings $B_{1}$ and $B_{2}$ are assumed to be equal and subjected to the radial force $F_{r}$, which is applied at the centre point of the pair. In addition to $F_{r}$, an axial thrust $K_{a}$, also acts on the system through a rigid centre shaft.

Geometrically, each bearing is defined by the following parameters: roller length, $L$; mean roller diameter, $d$ (measured at midlength of $L$ ); number of rollers, $Z$; pitch diameter of the roller set, $D$; contact angle, $\alpha$. The ISO 281 standard [22] specifies that this angle should measure the slope of the raceway without retaining ribs, which is normally the outer one (cup) as shown in Figure 2. Should the retaining rib be provided by the cup, the contact angle $\alpha$ should refer to the inner raceway (cone).

With reference to Figure 2, the paper seeks the set of bearing parameters $\{d, D, L, Z, \alpha\}$ which maximizes the rating life of the system for given forces $K_{a}$ and $F_{r}$. The search for the optimum will be based on the equations provided by the ISO 281 standard [22]. Although obtained explicitly for the particular bearing combination depicted in Figure 2 ( $\mathrm{O}$ arrangement with bearings removed from each other), the optimal solution presented will be applicable also to other combinations such as those shown in Figure $3(\mathrm{O}$ and $\mathrm{X}$ arrangements with removed or paired bearings).

\section{Theory}

\subsection{Basic dynamic radial load rating}

Subject to the conditions clarified below, the standard ISO 281 [22] gives the following field-tested expression for the dynamic radial load rating, $C$ (expressed in N) of tapered roller bearings with a single row of rollers

$$
C=1.1 f_{c}(L \cos \alpha)^{7 / 9} Z^{3 / 4} d^{29 / 27}
$$

where the lengths are expressed in $\mathrm{mm}$ and $f_{c}$ is a coefficient given in tabular form by ISO 281 and available analytically from the technical literature (see below). Equation (1) holds true if the bearing is built and installed under the following assumptions: (a) use of bearing steels with hardness $\mathrm{HRC} \geq 58$; (b) manufacture according to regular tolerances $[23,24]$ to enhance pressure uniformity 


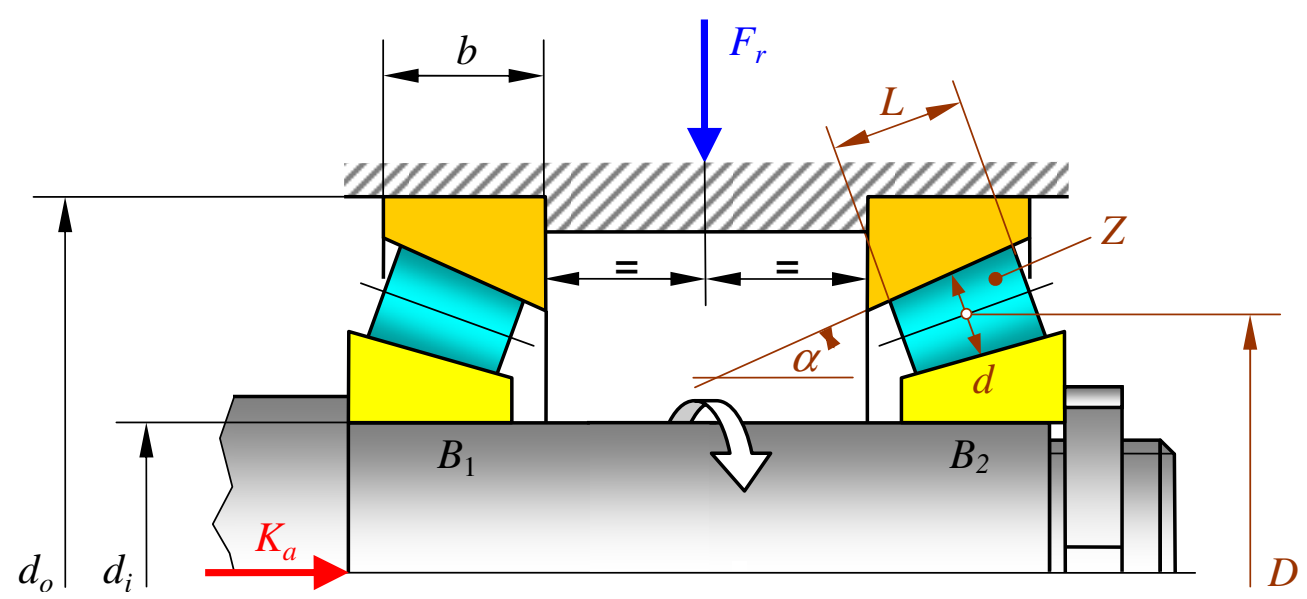

Fig. 2. Reference configuration of the bearing system with applied loads $\left(F_{r}\right.$ and $\left.K_{a}\right)$ and characteristic dimensions of the bearings.
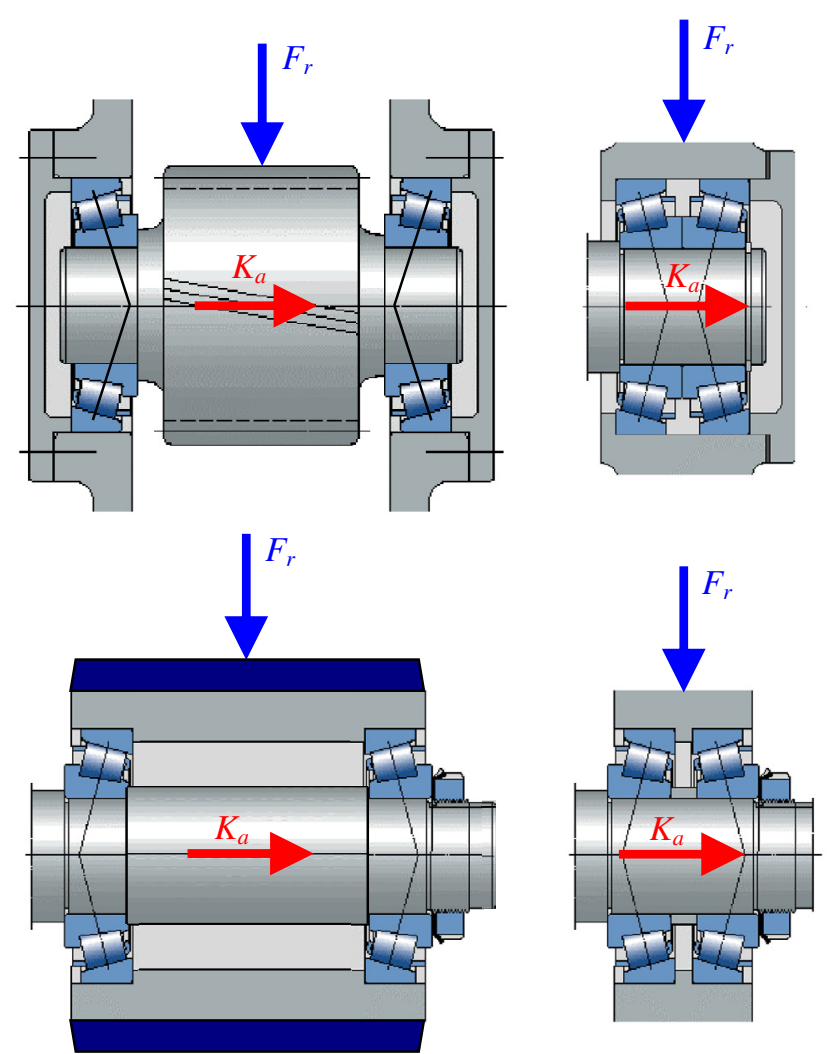

Fig. 3. Examples of bearing combinations to which the present theory is applicable: $\mathrm{X}$-arrangement (top); O-arrangement (bottom); not paired bearings (left); paired bearings (right).

over the roller length; (c) accurate guide of rollers with rounded ends to avoid pressure spikes at the edges [25];

(d) mounting onto stiff shafts and within rigid housings; (e) working temperature not higher than $150{ }^{\circ} \mathrm{C}$; (f) an angular load zone (i.e. the circumferential extent of the set of rollers that are in contact with both inner and outer raceways) of $180^{\circ}$. Significant deviations from these reference conditions can be accommodated either by applying correction factors available in reference [26] or by resorting to second-order methods [26,27] and specialized numerical tools [28] available to bearing manufacturers. The extent of the load zone (f) is particularly sensitive to the end play (i.e. axial clearance) with which the bearing pair is mounted and to axial displacements produced by the load (see further comments in Sect. 3.2).

Table 1 compares the dynamic load ratings predicted by Equation (1) with the actual load ratings of a selection of tapered roller bearings retrieved from the catalogue of a leading manufacturer (INA). The internal geometry of the bearings in Table 1 (properties from $\alpha$ to $Z$ ) were calculated starting from the catalogue properties (from $d_{i}$ to $Y$ ) using the method described in the Appendix.

By defining the filling ratio of the bearing, $\zeta$, the aspect ratio of the rollers, $\lambda$, and the pitch ratio, $\delta$, as follows

$$
\begin{aligned}
\zeta & =\frac{Z d}{\pi D} \\
\lambda & =\frac{L}{d} \\
\delta & =\frac{d}{D}
\end{aligned}
$$

Equation (1) becomes

$$
C=1.1 \pi^{3 / 4} \zeta^{3 / 4} \lambda^{7 / 9} D^{50 / 27} f_{c}(\delta \cos \alpha)^{119 / 108} \cos \alpha^{-35 / 108}
$$

Although the theoretical limits for the positive parameters $\zeta, \lambda$ and $\delta$ are $\zeta \leq 1, \lambda \geq 0$ and $\delta<1$, in practice the following ranges are generally observed: $0.5 \leq \zeta \leq 1$, $0.5 \leq \lambda \leq 2$ and $\delta \leq 0.2$.

From [26], the coefficient $f_{c}$ can be expressed in terms of $\alpha$ and $\delta$ as

$$
\begin{aligned}
f_{c} & =172.5\left\{1+\left[1.04\left(\frac{1-\delta \cos \alpha}{1+\delta \cos \alpha}\right)^{143 / 108}\right]^{9 / 2}\right\}^{-2 / 9} \\
& \times \frac{(\delta \cos \alpha)^{2 / 9}(1-\delta \cos \alpha)^{29 / 27}}{(1+\delta \cos \alpha)^{1 / 4}}
\end{aligned}
$$


Table 1. Comparison of predicted and catalogue dynamic load ratings for a selection of commercial tapered roller bearings (INA [32]).

\begin{tabular}{|c|c|c|c|c|c|c|c|c|c|c|c|}
\hline & \multicolumn{4}{|c|}{ Properties from INA catalogue } & \multicolumn{5}{|c|}{ Derived properties (see Appendix) } & \multicolumn{2}{|r|}{$C(\mathrm{kN})$} \\
\hline Bearing & $\begin{array}{c}d_{i} \\
(\mathrm{~mm})\end{array}$ & $\begin{array}{c}d_{o} \\
(\mathrm{~mm})\end{array}$ & $\begin{array}{c}b \\
(\mathrm{~mm})\end{array}$ & $\begin{array}{c}Y \\
(-)\end{array}$ & $\begin{array}{c}\alpha \\
\left({ }^{\circ}\right)\end{array}$ & $\begin{array}{c}d \\
\mathrm{r}(\mathrm{mm})\end{array}$ & $\begin{array}{c}D \\
(\mathrm{~mm})\end{array}$ & $\begin{array}{c}L \\
(\mathrm{~mm})\end{array}$ & $\begin{array}{c}Z \\
\mathrm{r}(-)\end{array}$ & INA & Equation (1) \\
\hline $30210-\mathrm{A}$ & 50 & 90 & 17 & 1.43 & 15.6 & 10 & 70 & 14.1 & 20 & 79 & 82 \\
\hline 30220-A & 100 & 180 & 29 & 1.43 & 15.6 & 20 & 140 & 24.1 & 20 & 250 & 263 \\
\hline $30230-\mathrm{A}$ & 150 & 270 & 38 & 1.38 & 16.2 & 30 & 210 & 31.6 & 20 & 465 & 501 \\
\hline $30310-\mathrm{A}$ & 50 & 110 & 23 & 1.74 & 12.9 & 15 & 80 & 18.9 & 15 & 130 & 131 \\
\hline 30320-A & 100 & 215 & 39 & 1.74 & 12.9 & 28.75 & 157.5 & 32 & 16 & 410 & 419 \\
\hline 30330-A & 150 & 320 & 55 & 1.74 & 12.9 & 42.5 & 235 & 45.1 & 16 & 800 & 832 \\
\hline 31310-A & 50 & 110 & 19 & 0.73 & 28.7 & 15 & 80 & 17.3 & 15 & 111 & 113 \\
\hline $31320-\mathrm{X}$ & 100 & 215 & 35 & 0.73 & 28.7 & 28.75 & 157.5 & 32 & 16 & 385 & 385 \\
\hline 31330-X & 150 & 320 & 50 & 0.73 & 28.7 & 42.5 & 235 & 45.7 & 16 & 790 & 790 \\
\hline T7FC050 & 50 & 105 & 22 & 0.69 & 30.1 & 13.75 & 77.5 & 20.3 & 16 & 127 & 127 \\
\hline T7FC070 & 70 & 140 & 27 & 0.69 & 30.1 & 17.5 & 105 & 25 & 17 & 208 & 208 \\
\hline T7FC095 & 95 & 180 & 33 & 0.69 & 30.1 & 21.25 & 137.5 & 30.5 & 19 & 325 & 325 \\
\hline
\end{tabular}

Letting

$$
\xi=\delta \cos \alpha
$$

combination of (5) and (6) gives

$$
\begin{aligned}
C= & 447.76\left(\zeta^{3 / 4} \lambda^{7 / 9} D^{50 / 27}\right) \\
\times & \frac{\delta^{35 / 108} \xi(1-\xi)^{29 / 27}}{(1+\xi)^{1 / 4}\left\{1+\left[1.04\left(\frac{1-\xi}{1+\xi}\right)^{143 / 108}\right]^{9 / 2}\right\}^{2 / 9}}
\end{aligned}
$$

\subsection{Dynamic equivalent radial load}

Let $F_{r s}=0.5 F_{r}$ be the radial load on the single bearing of the system in Figure 2 (remember that $F_{r}$ is assumed centred between $B_{1}$ and $\left.B_{2}\right)$ and $F_{a}$ the actual axial load on the most loaded of the two bearings (bearing $B_{1}$ in Fig. 2). Following ISO 281 [22], the dynamic equivalent radial load, $P$, acting on the most loaded roller bearing of the pair is

$$
P=X F_{r s}+Y F_{a}=X\left(0.5 F_{r}\right)+Y F_{a}
$$

where

$$
\begin{aligned}
& X=1 Y=0\left(\frac{F_{a}}{F_{r s}} \leqslant 1.5 \frac{\sin \alpha}{\cos \alpha}\right) \\
& X=0.4 Y=0.4 \frac{\cos \alpha}{\sin \alpha}\left(\frac{F_{a}}{F_{r s}}>1.5 \frac{\sin \alpha}{\cos \alpha}\right)
\end{aligned}
$$

Equation (9) does not account for narrow load zones (see Sect. 3.1) and uses a lower limit corresponding to a load zone of 180 degrees $\left(P=F_{r s}\right)$. Another implicit assumption behind Equation (9) is the absence of end moments acting on the shaft at the sections coupled with the bearings, which is consistent with the assumption of rigid shaft. For a flexible shaft, the radial loads on the two bearings would not necessarily be the same due to interdependence between radial displacements, tilt rotations and unequal load zones. Handling of these situations would necessarily call for the use of in-house tools developed by bearing manufacturers (e.g. [28]). Based on experience data from manufacturers catalogues, Harris [26] sets to $10^{-3}$ rad the maximum acceptable misalignment between shaft and roller bearings. This means that if the flexural rotation of the shaft relative to the housing is less than this limit, the bearing life is not shortened with respect to the standard equations used in this paper. Though the relative tilt of the shaft should take the stiffness of the bearings into account, conservatively it is more easily calculated by likening the shaft to and elastic beam supported by perfect hinges.

For a rigid shaft under the centre radial loading in Figure 2 and assuming neither end play nor axial preload on the system, the maximum axial force $F_{a}$ is given by the following universally accepted equation [29]

$F_{a}=K_{a}+0.5\left(\frac{F_{r s}}{Y}\right)=K_{a}+0.5\left(\frac{0.5 F_{r}}{Y}\right)=K_{a}+0.25 \frac{F_{r}}{Y}$

in which [29]

$$
Y=0.4 \frac{\cos \alpha}{\sin \alpha}
$$

Using professional tools, it can be shown that an axial assembly preload equal to $F_{a} / F_{r s} \approx 1.55$ would slightly improve the load carrying capacity of the bearing system with respect to the assumption of no end play. By contrast, a positive end play (clearance) or the axial displacement induced by the load itself would dramatically decrease the load zone of the secondary bearing (i.e. the bearing which does not support directly the external axial load, $B_{2}$ in Fig. 2), so that it could become the element of the pair which experiences the highest contact stresses. For this reason the end play should always be strictly controlled and positive values should be avoided whenever possible.

By virtue of (12) Equation (11) becomes

$$
F_{a}=K_{a}+0.625 F_{r} \frac{\sin \alpha}{\cos \alpha}
$$




$$
\left.L_{10}=\left(\frac{\zeta^{5 / 2} \lambda^{70 / 27} D^{500 / 81}}{F_{r}^{10 / 3}}\right) \cdot\left\{\frac{(1+\xi)^{1 / 4}\left\{1+\left[1.04\left(\frac{1-\xi}{1+\xi}\right)^{143 / 108}\right]^{9 / 2}\right\}^{2 / 9}}{\operatorname{MAX}\left(0.5 ; 0.45+0.4 \frac{\cos \alpha}{\sin \alpha} k\right)}\right\}^{\frac{10}{3}}\right\}
$$

from which, letting

$$
k=\frac{K_{a}}{F_{r}}
$$

the following relationship obtains

$$
\frac{F_{a}}{F_{r s}}=2 k+1.25 \frac{\sin \alpha}{\cos \alpha}
$$

By means of (10), (13) and (15) Equation (9) becomes

$$
P=\left\{\begin{array}{cc}
0.5 F_{r} & \left(k \leq 0.125 \frac{\sin \alpha}{\cos \alpha}\right) \\
\left(0.45+0.4 \frac{\cos \alpha}{\sin \alpha} k\right) F_{r} & \left(k>0.125 \frac{\sin \alpha}{\cos \alpha}\right)
\end{array}\right.
$$

Since the second relationship in (16) equals $0.5 F_{r}$ for $k=$ $0.125 \sin \alpha / \cos \alpha$, the equivalent radial load, $P$, can finally be cast as

$$
P=F_{r} \cdot \operatorname{MAX}\left\{\begin{array}{c}
0.5 \\
0.45+0.4 \frac{\cos \alpha}{\sin \alpha} k
\end{array}\right.
$$

\subsection{Basic rating life}

From ISO 281 [22] the basic rating life of the bearing in million revolutions, $L_{10}$, reads

$$
L_{10}=\left(\frac{C}{P}\right)^{10 / 3}
$$

By means of (8) and (17), Equation (18) becomes

which can be written as

$$
\text { see equation (19) above }
$$

$$
L_{10}=\phi\left(\omega_{10}\right)^{\frac{10}{3}}
$$

with

$$
\phi=\frac{\zeta^{5 / 2} \lambda^{70 / 27} D^{500 / 81}}{F_{r}^{10 / 3}}
$$

and

$$
\text { see equation (22) above }
$$

\section{Optimization}

\subsection{Free optimization}

Equation (19) shows that the basic rating life, $L_{10}$, is proportional to the functions $\phi$ and $\omega_{10}$. From (21) we see that, for given radial load $F_{r}$, function $\phi$ increases more than quadratically with the filling ratio, $\zeta$, and the aspect ratio, $\lambda$, and goes up with the sixth power of the pitch diameter, $D$. Function $\phi$ can be regarded as a control factor through which the basic rating life, $L_{10}$, can easily be made large at will by increasing the pitch diameter. Similarly, function $\omega_{10}$ in Equation (19) can be interpreted as the intrinsic rating life of the bearing system obtained when the filling ratio, the aspect ratio, the pitch diameter and the radial load assume unit value. From (22) we see that $\omega_{10}$ depends non-linearly on $\alpha, \delta$ and $k$ as shown by the three-dimensional charts in Figure 4.

Using $\omega_{10}$ as objective function, the free optimization problem can be stated as follows: Maximize $\omega_{10}(\boldsymbol{X})$, subject to $k=$ constant, with $\boldsymbol{X}=(\delta, \alpha)$. From Figure 4 it is seen that the maximum value of $\omega_{10}$ is always achieved for $\delta=\delta^{*}=1$. Entering $\delta=1$ in Equation (22) the optimal contact angles $\alpha^{*}$ can be obtained numerically for any load ratio $k$. Table 2 lists the optimal angles for load ratios in the range $0-1$. Figure 5 depicts the shape assumed by the optimal bearing for $k=1.0$.

\subsection{Constrained optimization}

The proportions of the optimal bearings implied by Table 2 and displayed in Figure 5 are too cumbersome to be of practical use. A more useful result is obtained by stating the optimization problem with a constraint on the pitch ratio as follows: Maximize $\omega_{10}(\boldsymbol{X})$, subject to $\delta \leq \delta_{\lim }$ and $k=$ constant, with $\boldsymbol{X}=(\alpha, \delta)$. This problem is plotted for several $k$ in Figure 6 , with the contour lines of $\omega_{10}$ drawn as a function of the contact angle, $\alpha$, and the pitch ratio, $\delta$. For any chosen load ratio $k$, the 
E. Dragoni: Mechanics \& Industry 18, 112 (2017)
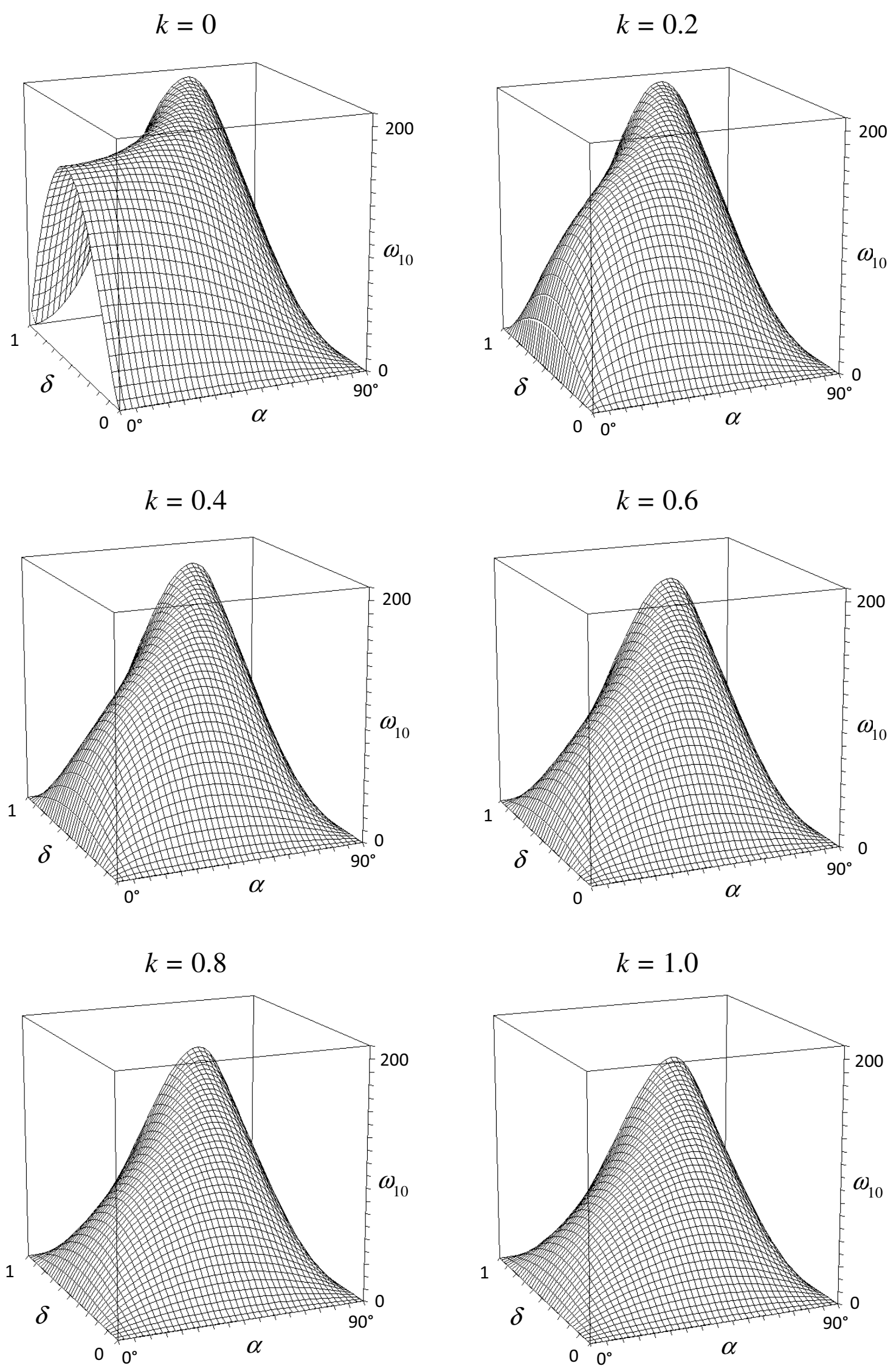

Fig. 4. Charts of the intrinsic rating life $\omega_{10}$ for a selection of load ratios $k$. 
Table 2. Global optimum contact angles $\left(\alpha^{*}\right)$ and pitch ratio $\left(\delta^{*}\right)$ for a range of load ratios $(k)$.

\begin{tabular}{cccc}
\hline$k$ & $\delta^{*}$ & $\alpha^{*}\left({ }^{\circ}\right)$ & $\omega_{10}^{*}$ \\
\hline 0 & 1 & 62.5 & 193.21 \\
0.1 & 1 & 62.5 & 193.21 \\
0.2 & 1 & 62.5 & 193.21 \\
0.3 & 1 & 65.0 & 189.61 \\
0.4 & 1 & 65.5 & 182.91 \\
0.5 & 1 & 66.0 & 176.79 \\
0.6 & 1 & 66.5 & 171.18 \\
0.7 & 1 & 67.0 & 166.01 \\
0.8 & 1 & 67.5 & 161.22 \\
0.9 & 1 & 68.0 & 156.76 \\
1.0 & 1 & 68.0 & 152.62 \\
\hline
\end{tabular}

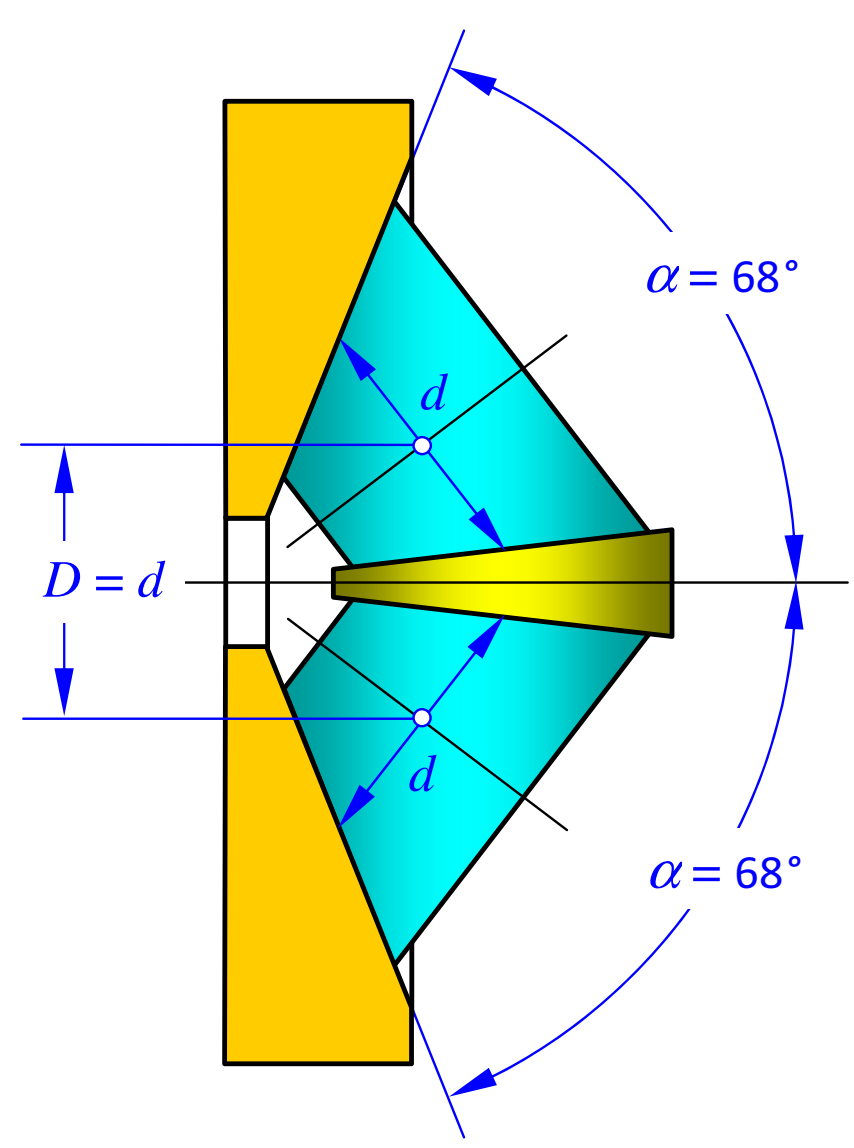

Fig. 5. Optimal bearing load ratio $k=1$ when no limits are put to the pitch ratio $\delta=d / D$.

coordinates of point $\boldsymbol{X}^{*}$ in Figure 6 provide the global optimal values $\alpha^{*}$ and $\delta^{*}$ listed in Table 2. Figure 6 shows that, for reasonable values of the limit pitch ratio (i.e. $\left.\delta_{\text {lim }} \leq 0.5\right)$, an optimal contact angle, $\alpha_{\delta}^{*}$, always exists, which depends on the limit pitch ratio itself. Take, for example the chart in Figure 6 for $k=0.8$ and assume $\delta \leq \delta_{\lim }=0.3$ meaning that only designs below line ab are feasible. The greatest value of $\omega_{10}$ that can be achieved in that region is obtained by moving on line $a b$ and sweeping the contact angle from $a$ to $b$ until the optimum point $\boldsymbol{M}^{*}$ is reached. Point $\boldsymbol{M}^{*}$ is defined as the tangent point between $a b$ itself and whichever contour line occurs to be touching the line $a b$. The abscissa of point $\boldsymbol{M}^{*}$ gives the optimal value $\alpha_{\delta}^{*}$ for the contact angle $\left(\alpha_{\delta}^{*} \approx 47.5^{\circ}\right.$ in this example). The contour line of $\omega_{10}$ passing through $\boldsymbol{M}^{*}$ gives the corresponding intrinsic rating life $\left(\omega_{10 \delta}^{*} \approx 60\right.$ in the example).

This constrained optimization can be performed systematically once and for all using Equation (22) for any combination of load ratios $k$ and pitch ratios $\delta$. For given $k$ and $\delta$, the intrinsic rating life $\omega_{10}$ in Equation (22) depends only on $\alpha$ and the optimum value $\alpha_{\delta}^{*}$ is easily found numerically. Optimal values of $\alpha_{\delta}^{*}$ and $\omega_{10 \delta}^{*}$ obtained in this way are reported in Table 3 for load ratios in the range $0 \leq k \leq 1.0$ and pitch ratios $0.05 \leq \delta \leq 0.25$ (the most likely to occur in practice). Optimal bearings for $\delta_{\lim }=0.2$ are shown in Figure 7 for $k=0\left(\alpha_{\delta}^{*}=0^{\circ}\right)$, $k=0.2\left(\alpha_{\delta}^{*}=31.5^{\circ}\right)$ and $k=0.4\left(\alpha_{\delta}^{*}=38^{\circ}\right)$. In marked contrast with the bearing proportions in Figure 5, awkward and hardly feasible, the designs in Figure 7 are sleek and technically viable.

The use of Table 3 for the optimal design of tapered roller bearings is easily performed as clarified by the following example. Assume that the bearing system in Figure 2 has to be designed for the loads $F_{r}=$ $600000 \mathrm{~N}$ and $K_{a}=180000 \mathrm{~N}$ with a rating life factor $L_{10}=50$ million revolutions. Calculating from (14) $k=K_{a} / F_{r}=600000 / 180000=0.3$ and assuming a limit pitch ratio $\delta_{\lim }=0.15$, Table 3 gives the optimal contact angle $\alpha_{\delta}^{*}=34^{\circ}$ and the optimal intrinsic rating life $\omega_{10 \delta}^{*}=38.3$. From Equation (20) the value $\phi_{\delta}^{*}=L_{10} /\left(\omega_{10 \delta}^{*}\right)^{10 / 3}=50 /(38.3)^{10 / 3} \approx 2.64 \times 10^{-4}$ is calculated, which, adopting a filling ratio $\zeta=0.8$ (Eq. (2)) and an aspect ratio $\lambda=1.5$ (Eq. (3)) and using (21), gives $D_{\delta}^{*}=\left(\phi_{\delta}^{*} F_{r}^{10 / 3} / \zeta^{5 / 2} \lambda^{70 / 27}\right)^{81 / 500}=$ $\left(0.00264 \times 600000^{10 / 3} / 0.8^{5 / 2} \times 1.5^{70 / 27}\right)^{81 / 500} \approx 320 \mathrm{~mm}$. From (4), (3) and (2), the optimal mean roller diameter, optimal roller length and optimal number of rollers are finally obtained as $d_{\delta}^{*}=\delta_{\lim } D_{\delta}^{*}=0.15 \times 320 \approx 48 \mathrm{~mm}$, $L_{\delta}^{*}=\lambda d_{\delta}^{*}=1.5 \times 48 \approx 72 \mathrm{~mm}, Z_{\delta}^{*}=\pi \zeta D_{\delta}^{*} / d_{\delta}^{*}=$ $\pi \times 0.8 \times 320 / 48 \approx 17$.

\section{Discussion}

\subsection{Review of the results}

Table 1 shows that, despite its simplicity, Equation (1) predicts quite accurately the dynamic load ratings of commercial tapered roller bearings. Within the wide range of dimensions and contact angles considered, the maximum error in Table 1 (see Appendix) is about 8\% (bearings 301230-A and T7FC095) while the average absolute error is just $4 \%$. Errors of the same order of magnitude were calculated by Dragoni [21] for the static load rating of the same bearings listed in Table 1 .

For the case of purely radial load $(k=0)$, Table 2 gives a global optimum solution with contact angle $\alpha^{*}=62.5^{\circ}$ and pitch ratio $\delta^{*}=1$, while the angle $\alpha^{*}=0$ would 
E. Dragoni: Mechanics \& Industry 18, 112 (2017)
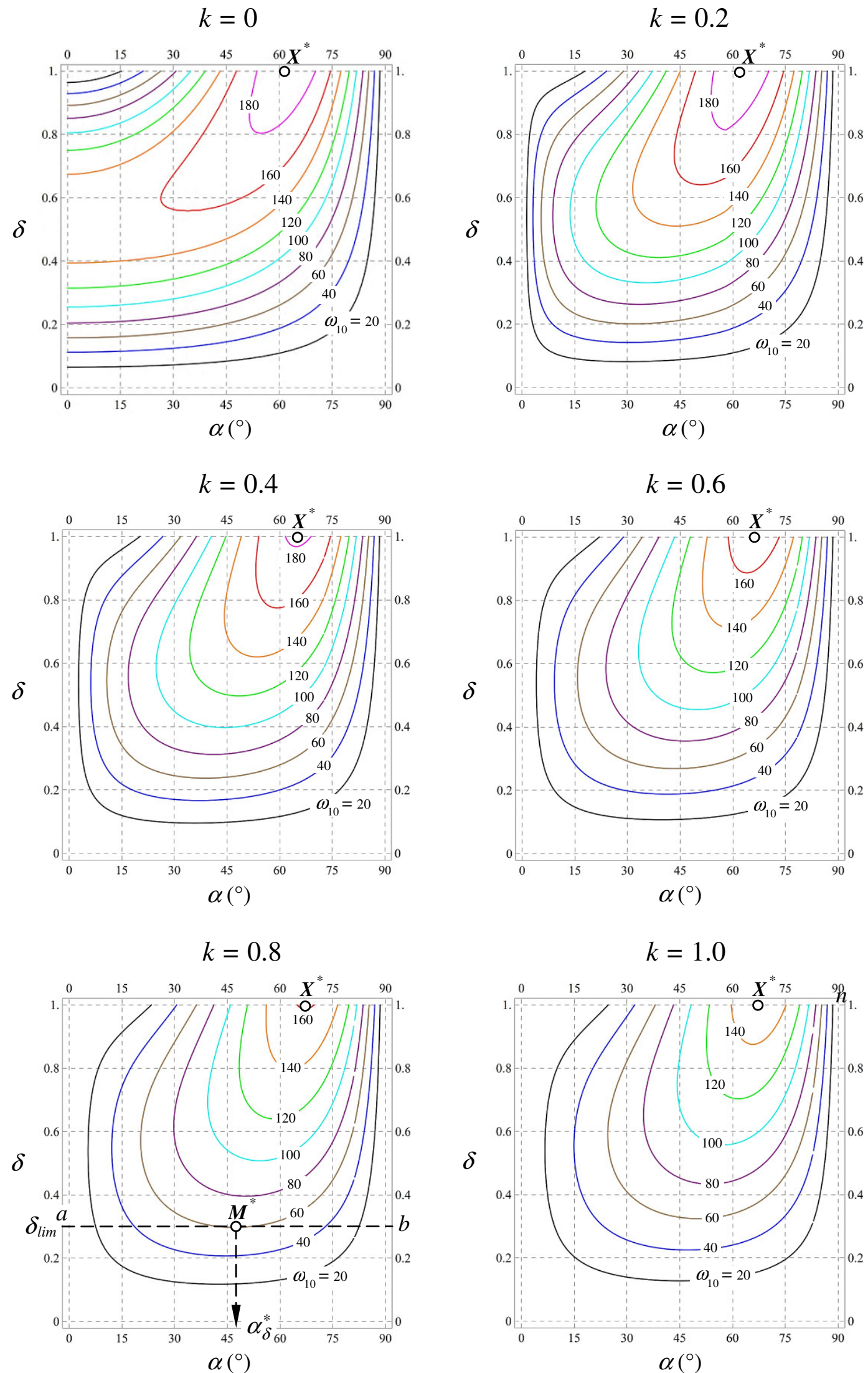

Fig. 6. Contour lines of the intrinsic rating life $\omega_{10}$ for a selection of load ratios $k$. 


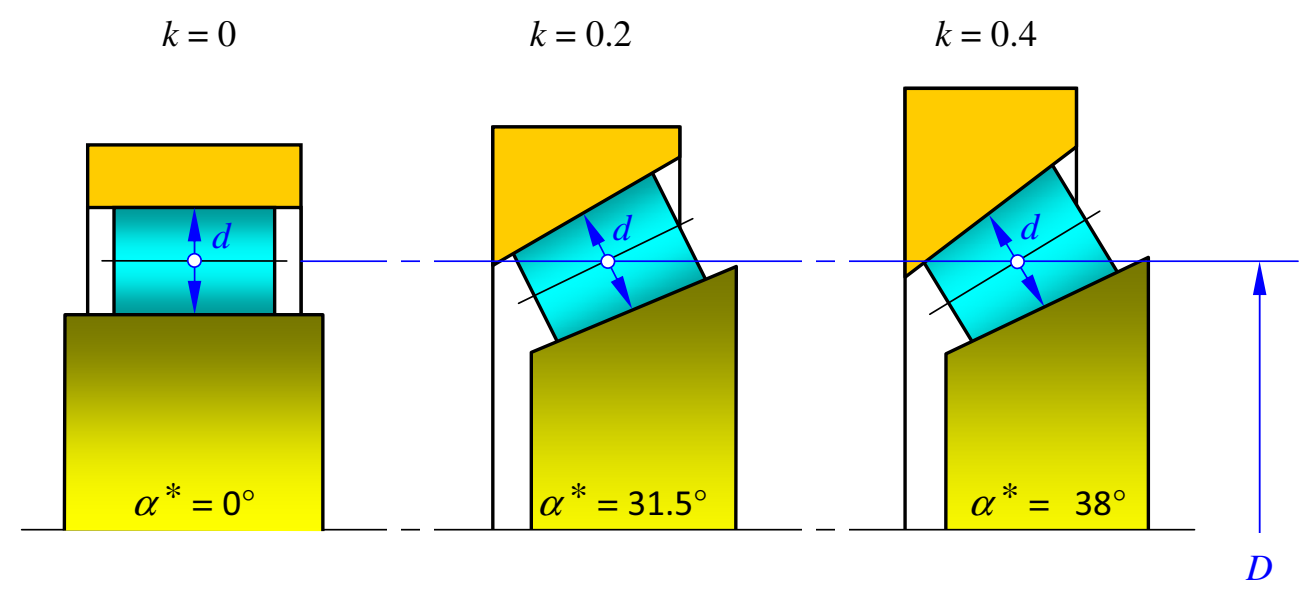

Fig. 7. Optimal bearings for different load ratios $k$ and limit pitch ratio $\delta_{\lim }=0.2$.

Table 3. Optimum values of contact angle $\left(\alpha_{\delta}^{*}\right)$ and corresponding intrinsic rating life $\left(\omega_{10 \delta}^{*}\right)$ for given load ratios $(k)$ and limit pitch ratios $\left(\delta_{\lim }\right)$.

\begin{tabular}{ccccccccccc}
\hline & \multicolumn{10}{c}{$\delta_{\lim }$} \\
\cline { 2 - 11 }$k$ & \multicolumn{2}{c}{0.05} & \multicolumn{2}{c}{0.1} & \multicolumn{1}{c}{0.15} & \multicolumn{2}{c}{0.2} & \multicolumn{2}{c}{0.25} \\
\cline { 2 - 11 } & $\alpha_{\delta}^{*}\left(^{\circ}\right)$ & $\omega_{10 \delta}^{*}$ & $\alpha_{\delta}^{*}\left(^{\circ}\right)$ & $\omega_{10 \delta}^{*}$ & $\alpha_{\delta}^{*}\left({ }^{\circ}\right)$ & $\omega_{10 \delta}^{*}$ & $\alpha_{\delta}^{*}\left(^{\circ}\right)$ & $\omega_{10 \delta}^{*}$ & $\alpha_{\delta}^{*}\left({ }^{\circ}\right)$ & $\omega^{*} 0 \delta$ \\
\hline 0 & 0 & 14.17 & 0 & 34.58 & 0 & 56.60 & 0 & 78.15 & 0 & 97.98 \\
0.1 & 24.0 & 12.01 & 24.5 & 29.43 & 25.5 & 48.55 & 26.0 & 67.69 & 27.5 & 85.88 \\
0.2 & 29.5 & 10.448 & 30.0 & 25.66 & 30.5 & 42.48 & 31.5 & 59.49 & 33.0 & 75.86 \\
0.3 & 33.0 & 9.38 & 33.5 & 23.08 & 34.0 & 38.30 & 35.5 & 53.80 & 36.5 & 68.86 \\
0.4 & 35.5 & 8.58 & 36.0 & 21.12 & 37.0 & 35.11 & 38.0 & 49.45 & 39.5 & 63.47 \\
0.5 & 37.5 & 7.93 & 38.0 & 19.56 & 39.0 & 32.56 & 40.0 & 45.94 & 41.5 & 59.11 \\
0.6 & 39.0 & 7.40 & 39.5 & 18.26 & 40.5 & 30.44 & 41.5 & 43.02 & 43.0 & 55.46 \\
0.7 & 40.5 & 6.95 & 41.0 & 17.16 & 42.0 & 28.63 & 43.0 & 40.53 & 44.5 & 52,34 \\
0.8 & 42.0 & 6.56 & 42.5 & 16.21 & 43.5 & 27.07 & 44.5 & 38.374 & 46.0 & 49.63 \\
0.9 & 43.0 & 6.22 & 43.5 & 15.38 & 44.5 & 25.71 & 45.5 & 36.48 & 47.0 & 47.24 \\
1.0 & 44.0 & 5.92 & 44.5 & 14.64 & 45.5 & 24.49 & 46.5 & 34.80 & 48.0 & 45.12 \\
\hline
\end{tabular}

have been expected. For $k=0$ and $\alpha=0$, Figure 4 shows that the intrinsic rating life achieves a maximum for $\delta=1$, which means cylindrical rollers of diameter, $d$, equal to one half of the pitch diameter, $D$. This result coincides with the global optimal proportions reported by Dragoni [20] for the specific category of radial cylindrical roller bearings. For increasing axial loads $(k>0)$, the global optima for the pitch ratio listed in Table 2 become more and more unlikely for real-life applications (see Fig. 5 for $k=1$ ). The reason why the theoretical optimization tends to these quite odd shapes is perhaps imputable to the fact that the empirical expression (1) was developed to fit the experimental behaviour of bearings with pitch ratios much lower than 0.5 as commonly encountered in practice (for example, the pitch ratios of the twelve commercial bearings in Tab. 1 range from 0.14 to 0.19$)$.

Table 3, developed to take into account realistic geometric constraint on $\delta\left(\delta \leq \delta_{\lim }=0.05 \ldots 0.25\right)$, shows that the optimum contact angle, $\alpha_{\delta}^{*}$, depends both on the load ratio, $k$, and the limit pitch ratio, $\delta_{\text {lim }}$. However, while the effect of the load ratio is strong (with $\alpha_{\delta}^{*}$ increasing monotonically with $k$ ), the dependence on the pitch ratio is weak (on passing from $\delta_{\lim }=0.05$ to $\delta_{\lim }=0.25$, the average increase of the optimal contact angle is about $11 \%$, with a peak value of $15 \%$ for $k=0.6$ ). For $k=0.2$, the optimal contact angle in Table 3 is around $30^{\circ}$, which is the highest contact angle prescribed by the ISO 355 standard [30] and offered by most bearing manufacturers (see Tab. 1). For load ratios greater than 0.2 , the optimal contact angle exceeds $30^{\circ}$ and reaches the optimum value of about $45^{\circ}$ for $k=1$. In this range of operation, special supplies $[31,32]$ or custom constructions are needed to achieve the maximum load capacity.

Figure 8 compares the optimal angles from Table 3 (optimization for dynamic loading) with the corresponding optima (Table 3 of [21]) for the static loading of taper roller bearings. Figure 8 shows that the optimal dynamic contact angles are generally higher than their static counterparts for equal $\delta_{\text {lim }}$. This is especially true for low load ratios $k$ (for example, for $k=0.1$ the optimal dynamic contact angle in Table 3 ranges from $24^{\circ}$ to $27.5^{\circ}$, contrasted with an optimal static angle of $11^{\circ}$ from [21]) while the difference is much reduced for $k$ equal to or higher than 0.3 (for $k=0.3$, the dynamic optimal angles in Table 3 range from $33^{\circ}$ to $36^{\circ}$, compared with optimal static angles ranging from $29.5^{\circ}$ to $30.0^{\circ}$. For $k=1$ and $\delta_{\lim }=0.25$, the optimal dynamic angle of $48^{\circ}$ compares 


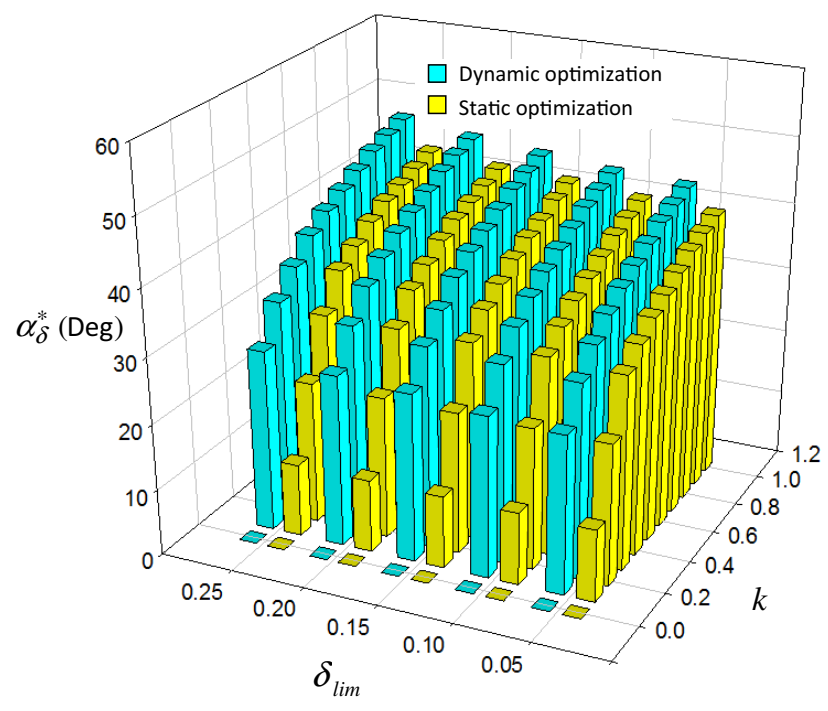

Fig. 8. Comparison of optimal contact angles for the static case (Table 3 of [21]) and the present dynamic case.

with the optimal static angle of $43.5^{\circ}$ ). This means that, when the axial load is significant, the optimization is robust and applies to the bearing in itself, regardless of the nature of the loading (static or dynamic) involved.

Walker [16] argues that for low-speed roller bearings loaded under prevailing axial thrusts, the optimal cone angle is about $40^{\circ}$ as predicted by Table 3 for $k \geq 0.6$. Conversely, for $k=0$, purely radial load, Table 3 correctly predicts $\alpha^{*}=0^{\circ}$, implying the adoption of bearings with cylindrical rollers.

Table 3 also shows that the optimal intrinsic rating life, $\omega_{10 \delta}^{*}$, rapidly increases with the limit pitch ratio, $\delta_{\lim }$, and decreases with the load ratio, $k$. The increase of $\omega_{10 \delta}^{*}$ with $\delta_{\lim }$ is a consequence of the marked gradient of the surfaces of $\omega_{10}$ in Figure 4 (confirmed by the density of the contour lines in Fig. 6) for pitch ratios in the range $0 \leq \delta \leq 0.5$. The decrease of $\omega_{10 \delta}^{*}$ with $k$ is due to the fact that, given the radial force $F_{r}$ in Figure 2, an increase of the load ratio $k$ implies a greater total force on the bearing system with respect to the condition of pure radial loading.

With reference to the numerical example at the end of the Section Constrained optimization, it is easily verified that substituting the design data $F_{r}=600 \mathrm{kN}$ and $k=0.3$, together with the optimal results $\alpha_{\delta}^{*}=34^{\circ}$, $d_{\delta}^{*}=48 \mathrm{~mm}, L_{\delta}^{*}=72 \mathrm{~mm}, Z_{\delta}^{*}=17$ for $F_{r}, k, \alpha, d, L$ and $Z$, in Equations (17) and (1), gives $P=376.7 \mathrm{kN}$ and $C=1228 \mathrm{kN}$, respectively. These values imply a rating life $L_{10}=(C / P)^{10 / 3}=(1228 / 376.7)^{10 / 3} \approx 51.4$ million revolutions, slightly greater than the design value of 50 million. This small difference is due to roundoff of the variables involved in the calculations, especially as regards to the optimum number of rollers $Z_{\delta}^{*}$ (the exact value 16.76 was rounded to 17 in the example).

\subsection{Limitations of the model}

The present optimization is built on the assumption that the critical bearing of the pair is the one that directly supports the axial load ( $B_{1}$ in Fig. 2). This is the natural consequence of using Equation (9) for calculating the maximum equivalent load on the system. Though this approach is coherent with the design formulae present in the manufacturers' catalogues, it has limitations when the bearing pair is mounted with end play (axial clearance) or undergoes large axial deformations under load. A large axial load induces a large axial displacement which causes a very narrow load zone in the second bearing $\left(B_{2}\right.$ in Fig. 2, not considered here), hence generating risk of large roller-race load and pressure. Under these unfavourable working conditions, the second bearing may thus become the critical element, which is not optimized here. In this case, the full answer to the problem cannot be obtained with the present analytical model and requires numerical tools considering equilibrium and compatibility (deformations) of bearings, shaft and housing as a whole.

In practical terms, the optimal design described in this paper strictly holds true when the bearing system is assembled with a light preload that would compensate the axial displacement induced in the secondary bearing by the externally applied load. This is certainly a limitation, but not a prohibitive one since preloading of tapered roller bearings is a common procedure for the many advantages it brings about such as (a) increase of the stiffness, (b) noise reduction, (c) improved rotational precision, (d) wear compensation, and (e) longer life.

\section{Conclusions}

Using the empirical relationships provided by the ISO 281 standard, the internal dimensions of tapered roller bearings are optimized for maximum dynamic capacity. The bearing system investigated comprises two identical bearings undergoing whatever combination of radial and axial forces. Assuming that the radial force is applied at equal distance from the bearings of the pair and that neither end play (axial clearance) nor abnormal preloading affect the assembly, the optimization process leads to the following general results:

- the rating life decreases approximately with the third power of the applied radial force;

- the rating life increases more than quadratically with the filling ratio (number of rollers divided by the maximum number which can fill the bearing) and with the aspect ratio (ratio of roller length to mean roller diameter) and goes up with the sixth power of the pitch diameter of the roller set;

- given the ratio of axial to radial force, global optima exist for the contact angle and the pitch ratio (ratio of roller diameter to pitch diameter) which maximize the rating life;

- the bearing proportions at the global optima are too cumbersome (contact angles greater than $60^{\circ}$, pitch ratios equal to 1.0) to be used in practice; 
Table A.1. Relations between catalogue data $\left(Y, d_{i}, d_{o}, b\right)$ and internal bearing properties $(\alpha, d, D, L, Z)$.

\begin{tabular}{cccc}
\hline Internal property & Relationship & Numerical values & Source \\
\hline$\alpha$ & $=\arctan \left(\frac{0.4}{Y}\right)$ & - & Equation (12) \\
$d$ & $=q_{1}\left(d_{o}-d_{i}\right)$ & $q_{1}=0.25$ & Textbook [33] \\
$D$ & $=q_{2}\left(d_{o}+d_{i}\right)$ & $q_{2}=0.5$ & Assumption \\
$L$ & $=q_{3} \frac{b}{\cos \alpha}$ & $q_{3}=0.8$ & Assumption \\
& $=q_{4} \frac{\left(d_{o}+d_{i}\right)}{d}$ & $q_{4}=1.45$ & Textbook [33] \\
\hline
\end{tabular}

- if the pitch ratio is constrained below reasonable limits $(\leq 0.25)$, an optimal contact angle exists which maximizes the rating life, regardless of the actual size and proportions of the bearing;

- the results of the optimization are conveniently summarized by a general table and a few simple equations that can be followed step-by-step to design the optimal bearing that suits any given application;

- the optimization procedure can either be used to design custom-made bearings (thus exploiting the geometrical freedom to the full) or to pick from the manufacturers' catalogues the bearing with the best contact angle for any assigned loading.

\section{Declaration of conflicting interests}

The author declares that there is no conflict of interest.

\section{Funding}

This research received no specific grant from any funding agency in the public, commercial, or not-for-profit sectors.

\section{Appendix (Internal dimensions of tapered roller bearings)}

The internal dimensions of rolling bearings are proprietary data which the manufacturers do not provide in their catalogues. However, starting from the external dimensions of the bearings $\left(d_{i}, d_{o}, b\right.$ in Fig. 2) and the dynamic coefficient, $Y$, available from the catalogues, the internal properties ( $\alpha, d, D, L, Z$ in Fig. 2) can be calculated within $10-15 \%$ of the true value. Table A.1 shows how the internal properties displayed in Table 1 were obtained step-by-step from the catalogue data using elementary geometrical considerations and characteristic proportions available from technical textbooks [33].

\section{References}

[1] A. Seireg, A survey of optimisation of mechanical design, J. Eng. Ind. TASME 94 (1972) 495-499

[2] A.A. Seireg, J. Rodriguez, Optimizing the shape of mechanical elements and structures, CRC Press, Cleveland, $\mathrm{OH}, 1997$

[3] J. Ahluwalia, S.K. Gupta, V.P. Agrawal, Computeraided optimum selection of roller bearings, Comput.-Aid. Design 25 (1993) 493-499

[4] H. Aramaki, Rolling Bearing Analysis Program Package "BRAIN", NSK Technical Journal Motion \& Control, 1997, No. 3, pp. 15-24

[5] D.-H. Choi, K.-C. Yoon, A Design Method of an Automotive Wheel-Bearing Unit With Discrete Design Variables Using Genetic Algorithms, J. Tribol. TASME 123 (2001) 181-187

[6] K. Kalita, R. Tiwari, S.K. Kakoty, Multi-objective optimisation in rolling element bearing system design, In: Proceedings of the Int. Conf. on Optimisation (SIGOPT 2002), Lambrecht, Germany, 17-22 February 2002

[7] I. Chakraborty, V. Kumar, S.B. Nair, R. Tiwari, Rolling element bearing design through genetic algorithms, Eng. Optimiz. 35 (2003) 649-659

[8] B.R. Rao, R. Tiwari, Optimum design of rolling element bearings using genetic algorithms, Mech. Mach. Theory 42 (2007) 233-250

[9] S. Gupta, R. Tiwari, S.B. Nair, Multi-objective design optimisation of rolling bearings using genetic algorithms, Mech. Mach. Theory 42 (2007) 1418-1443

[10] V. Savsani, R.V. Rao, D.P. Vakharia, Multi-objective design optimization of ball bearings using a modified particle swarm optimization technique, Int. J. Des. Eng. 1 (2008) 412-433

[11] Y. Wei, R. Chengzu, Optimal design of high speed angular contact ball bearing using a multiobjective evolution algorithm, In: Proceedings of the IEEE Int. Conf. on Computing, Control and Industrial Engineering (CCIE 2010), Wuhan, China, 5-6 June 2010

[12] K.S. Kumar, R. Tiwari, R.S. Reddy, Development of an optimum design methodology of cylindrical roller bearings using genetic algorithms, Int. J. Comput. Meth. Eng. Sci. Mech. 9 (2008) 321-341

[13] K.S. Kumar, R. Tiwari, V.V.N. Prasad, An Optimum Design of Crowned Cylindrical Roller Bearings Using Genetic Algorithms, J. Mech. Des. TASME 131 (2009) 051011-1-051011-14

[14] R.J. Parker, S.I. Pinel, H.R. Signer, Performance of Computer-Optimized Tapered-Roller Bearings to 2.4 Million DN, J. Tribol. TASME 103 (1981) 13-20 
[15] N. Chaturbhuj, S.B. Nair, R. Tiwari, Design optimization for tapered roller bearings using genetic algorithms, In: Proceedings of the Int. Conf. on Artificial Intelligence (IC-AI 03 2003), Las Vegas, Nevada, 23-26 June 2003, CSREA Press, ISBN 1-932415-12-2, Vol. 1, pp. $421-427$.

[16] B. Walker, High Speed Tapered Roller Bearing Optimization, MS Thesis, Rensselaer Polytechnic Institute, Hartford, Connecticut, April 2008

[17] Z. Wang, L. Meng, H. Wensi, E. Zhang, Optimal Design of Parameters for Four Column Tapered Roller Bearing, Appl. Mech. Mater. 63-64 (2011) 201-204

[18] R. Tiwari, K.K. Sunil, R.S. Reddy, An Optimal Design Methodology of Tapered Roller Bearings Using Genetic Algorithms, Int. J. Comp. Meth. Eng. Sci. Mech. 13 (2012) 108-127

[19] R. Tiwari, R. Chandran, Thermal Based Optimum Design of Tapered Roller Bearing Through Evolutionary Algorithm, In: Proceedings of the ASME 2013 Gas Turbine India Conference, Bangalore, Karnataka, India, 5-6 December 2013 (paper no. GTINDIA2013-3792)

[20] E. Dragoni, Optimal design of radial cylindrical roller bearings for maximum load-carrying capacity, Proc. IMechE, Part C: J. Mech. Eng. Sci. 227 (2013) 2393-2401

[21] E. Dragoni, Optimal Design of Paired Tapered Roller Bearings Under Centred Radial and Axial Static Loads, Mech Ind, (in press)

[22] ISO 281, Rolling bearings - Dynamic load ratings and rating life, 2007
[23] H. Wiesner, Rolling bearings TC4 meets GPS TC213, Evolution 19 (2012) 24-28

[24] ISO 492, Rolling bearings - Radial bearings - Tolerances, 2002

[25] K.L. Johnson, Contact Mechanics, Cambridge University Press, Cambridge, UK, 1985

[26] T.A. Harris, Rolling Bearing Analysis, 4th edition John Wiley \& Sons, New York, 2000

[27] NSK, Bearing internal load distribution and displacement, http://www.jp.nsk.com/app01/en/ctrg/ index.cgi?gr=dn $\backslash \&$ pno=nsk_cat_e728g_5 (access date 29/5/2015)

[28] INA, BEARINX-online Shaft Calculation, http:// www.ina.de/content.ina.de/en/products_services/ calculating/bearinxonline/bearinx_online.jsp (access date 29/5/2015)

[29] G. Niemann, H. Winter, B.-R. Höhn, Maschinenelemente, Springer, Germany, Berlin, 2005, Vol. I

[30] ISO 355, Rolling bearings - Tapered roller bearings Boundary dimensions and series designations, 2007

[31] TIMKEN, Tapered Roller Bearing Catalogue, http:// www. timken. com/en-us/products/Pages/Catalogs . aspx (access date 29/5/2015)

[32] INA, Tapered Roller Bearing Catalogue, http://www. ina.de/content.ina.de/de/products_services/ rotativ_products/tapered_roller_bearings/ tapered_roller_bearings.jsp (access date 29/5/2015)

[33] G. Niemann, Maschinenelemente, Springer, Berlin, Germany, 1981, Vol. I 